\title{
AN SIRS EPIDEMIC MODEL OF JAPANESE ENCEPHALITIS
}

\author{
B.B. MUKHOPADHYAY \\ Department of Community Medicine \\ Burdwan Medical College, Burdwan 713104 \\ West Bengal, India \\ and \\ P.K. TAPASWI \\ Embryology Unit \\ Indian Statistical Institute \\ Calcutta 700 035, India
}

(Received October 3, 1991 and in revised form October 5, 1992)

\begin{abstract}
An epidemiological model of the dynamics of Japanese Encephalitis (J.E.) spread coupling the SIRS (Susceptible/Infected/Removal/Susceptible) models of J.E. spread in the reservoir population and in the human population has been proposed. The basic reproductive rate $R(0)$ in the coupled system has been worked out. Using Aron's results (cf. [1] and [2]), it has been observed that the disease-free system is stable in this coupled system also, if $R(0)$ is less than unity, and if $R(0)$ is greater than unity, the disease-free system is unstable and there exists a unique stable endemic equilibrium.

The model also shows that in contrast to Aron's observations, loss of immunity is independent of the rate of exposure to the disease. This observation sheds light on the control measure of J.E. by vaccination. Passive immunization, i.e., administration of antibody at recurrent intervals is the correct method of vaccination to eradicate the disease.
\end{abstract}

KEY WORDS AND PHRASES. SIRS model, Japanese encephalitis, basic reproduction rate, stability analysis, control measure.

1980 AMS SUBJECT CLASSIFICATION CODE. 92A17.

\section{INTRODUCTION.}

Japanese Encephalitis (J.E.) is a mosquito borne disease where infection is transmitted from reservoir population (pig, cattle, equine, bird, etc.) to susceptible human population through a particular species of mosquito (Culex Vishnui). Man is the dead end of infection and as such harboring of infection from man to man is not possible. Immunity in both reservoir and human populations appear to be sustained by continual exposure. The present paper investigates into the epidemiological effect of boosting immunity in J.E. and the qualitative dynamics of the epidemiological model. Since transmission from man to man does not occur, man does not act as a carrier in J.E. On the other hand, the reservoir population (infective and immune carriers) not exhibiting the clinical symptoms, act as active hosts permitting transmission to both animal and 
human susceptibles. Thus in J.E. some proportion of immune reservoir population also acts as infective (active carrier), and the constraint that the degree of infectivity reduces with the increase of immunity status in them has been incorporated in this model following Aron (cf. [1] and [2]).

\section{MATHEMATICAL MODEL OF J.E.}

Both human and reservoir populations are classified into three categories, namely susceptible, infected and removal classes. Let $x_{1}, x_{2}$ and $x_{3}$ be the proportion of susceptibles, infected and removed respectively in human population and $y_{1}, y_{2}$ and $y_{3}$ be those for reservoir populations. The removed class includes both recovered (immune) and dead by J.E. The dynamical system representing the epidemic spread in human and reservoir populations are then given by the following rate equations

$$
\begin{aligned}
& \frac{d x_{1}}{d t}=\mu_{1}-\mu_{1} x_{1}-h_{1} x_{1}+f_{1} x_{3} \\
& \frac{d x_{2}}{d t}=h_{1} x_{1}-\mu_{1} x_{2}-\gamma_{1} x_{2} \\
& \frac{d x_{3}}{d t}=\gamma_{1} x_{2}-\mu_{1} x_{3}-f_{1} x_{3} \\
& \frac{d y_{1}}{d t}=\mu_{2}-h_{2} y_{1}-\mu_{2} y_{1}+f_{2} y_{3} \\
& \frac{d y_{2}}{d t}=h_{2} y_{1}-\mu_{2} y_{2}-\gamma_{2} y_{2} \\
& \frac{d y_{3}}{d t}=\gamma_{2} y_{2}-\mu_{2} y_{3}-f_{2} y_{3}
\end{aligned}
$$

where

and

$$
\begin{aligned}
& x_{1}(t)+x_{2}(t)+x_{3}(t)=1 \\
& y_{1}(t)+y_{2}(t)+y_{3}(t)=1 \\
& h_{1}=\beta_{1}\left(k_{1}^{\prime} y_{1}+k_{1}^{\prime \prime} y_{3}\right) x_{1}
\end{aligned}
$$

is the effective exposure rate for man and

$$
h_{2}=\beta_{2}\left(k_{2}^{\prime} y_{2}+k_{2}^{\prime \prime} y_{3}\right)
$$

is the exposure rate in reservoir population

$$
0 \leq k_{1}^{\prime \prime} \leq k_{1}^{\prime} \leq 1 ; \quad 0 \leq k_{2}^{\prime \prime} \leq k_{2}^{\prime} \leq 1 .
$$

$\mu_{1}$ is the birth and death rate in man, so that the population size remains the same. $\beta_{1}$ is the rate at which the human susceptibles become sick by mass action contact between susceptible man and infective reservoir populations. $k_{1}^{\prime}$ and $k_{1}^{\prime \prime}$ are the proportions of infected and immune reservoir respectively who are infective to man. Sick human individuals enter the removal class (recovered and dead) at the rate $\gamma_{1}$ and immune individuals (i.e., the recovered portion of the removal class $x_{3}$ ) become susceptibles at the rate $f_{1}, f_{1}$ being a function of $h_{1}$, and as derived by Aron [1] is

$$
f_{1}\left(h_{1}\right)=\frac{\left(h_{1}+\mu_{1}\right) e^{-\left(h_{1}+\mu_{1}\right) \tau_{1}}}{1-e^{-\left(h_{1}+\mu_{1}\right) \tau_{1}}}
$$

$\tau_{1}$ being the unit of years in which immunity in man lasts unless reexposure occurs during that time interval. The function $f_{1}\left(h_{1}\right)$ is a monotonically decreasing function of $h_{1}$ (cf. [1]; see also 
[2]). The rate constants $\mu_{2}, \beta_{2}$ and $\gamma_{2}$ in reservoir population stand for the same connotations as for the corresponding rate constants in man. $k_{2}^{\prime}$ and $k_{2}^{\prime \prime}$ are the proportions of infected and immune reservoirs respectively who are infective to the reservoir population. We also have similar expression for $f_{2}\left(h_{2}\right)$ as in $(2.4)$,

$$
f_{2}\left(h_{2}\right)=\frac{\left(h_{2}+\mu_{2}\right) e^{-\left(h_{2}+\mu_{2}\right) \tau_{2}}}{1-e^{-\left(h_{2}+\mu_{2}\right) \tau_{2}}}
$$

$\tau_{2}$ is the unit of years in which immunity in the reservoir population lasts unless reexposure occurs during that time period.

It may be noted that $h_{1}$ involves second order term whereas $h_{2}$ involves first order term only. this is apparent from the mode of transmission of the disease which is unlike malaria. Transmission of J.E. in man takes place by the interaction of the susceptible human populations and infected or carrier reservoir population (mediated by vector population), whereas transmission of J.E. in reservoir population occurs by direct contact (through vector) amongst themselves.

Considering that the proportion of infective reservoir population effective for infecting the susceptible man is usually higher than or at most equal to that effective for infecting the reservoir population itself, we can assume $k_{1}^{\prime} \geq k_{2}^{\prime}$ and $k_{1}^{\prime \prime} \geq k_{2}^{\prime \prime}$. Moreover, because of persistently boosted acquired immunity in the reservoir population, the transmission rates $\beta_{1}$ and $\beta_{2}$ satisfy the inequality relation, $\beta_{1} \geq \beta_{2}$. Hence

$$
\beta_{1}\left(k_{1}^{\prime} y_{2}+k_{1}^{\prime \prime} y_{3}\right) \geq \beta_{2}\left(k_{2}^{\prime} y_{2}+k_{2}^{\prime \prime} y_{3}\right) .
$$

Again, since $h_{1}$, the exposure rate in man can at most be equal to $h_{2}$, the exposure rate in the reservoir population, and also $x_{1} \leq 1$, we can take as a particular case

$$
\begin{gathered}
\beta_{1}\left(k_{1}^{\prime} y_{2}+k_{1}^{\prime \prime} y_{3}\right) x_{1}=\beta_{2}\left(k_{2}^{\prime} y_{2}+k_{2}^{\prime \prime} y_{3}\right) \\
\text { i.e., } h_{1}=h_{2}=h .
\end{gathered}
$$

When equality holds in (2.6) we have $x_{1}=1$, under which circumstances the disease process cannot start at all and hence, a requisite condition for the spread of the epidemic is that the inequality condition in (2.6) must be satisfied.

\section{EQUILIBRIA OF MODEL.}

For a particular $h$, the equilibrium values of $y_{2}, y_{3}$ and $x_{1}, x_{2}, x_{3}$ are

$$
\begin{aligned}
& y_{2}=\frac{h\left(\mu_{2}+f_{2}(h)\right)}{\left(\mu_{2}+\gamma_{2}\right)\left(\mu_{2}+f_{2}(h)\right)+h\left(\mu_{2}+\gamma_{2}+f_{2}(h)\right)} \\
& y_{3}=\frac{h \gamma_{2}}{\left(\mu_{2}+\gamma_{2}\right)\left(\mu_{2}+f_{2}(h)\right)+h\left(\mu_{2}+\gamma_{2}+f_{2}(h)\right)} \\
& x_{1}=\frac{\left(\mu_{1}+f_{1}(h)\right)\left(\mu_{1}+\gamma_{1}\right)}{\left(\mu_{1}+\gamma_{1}\right)\left(\mu_{1}+f_{1}(h)+h\right)+h f_{1}(h)} \\
& x_{2}=\frac{h\left(\mu_{1}+f_{1}(h)\right)}{\left(\mu_{1}+\gamma_{1}\right)\left(\mu_{1}+f_{1}(h)+h\right)+h f_{1}(h)} \\
& x_{3}=\frac{\gamma_{1} h}{\left(\mu_{1}+\gamma_{1}\right)\left(\mu_{1}+f_{1}(h)+h\right)+h f_{1}(h)}
\end{aligned}
$$

and therefore 


$$
\begin{aligned}
\left(k_{1}^{\prime} y_{2}+k_{1}^{\prime \prime} y_{3}\right) x_{1}= & \frac{h\left\{k_{1}^{\prime}\left(\mu_{2}+f_{2}(h)\right)+k_{1}^{\prime \prime} \gamma_{2}\right\}\left(\mu_{1}+\gamma_{1}\right)\left(\mu_{1}+f_{1}(h)\right)}{\left\{\left(\mu_{2}+\gamma_{2}\right)\left(\mu_{2}+f_{2}+h\right)+h f_{2}(h)\right\}\left\{\left(\mu_{1}+\gamma_{1}\right)\left(\mu_{1}+f_{1}(h)+h\right)+h f_{1}(h)\right\}} \\
& =\text { effective reservoir of infection for man. }
\end{aligned}
$$

Again from (2.2) and (2.6) we have

$$
\left(k_{1}^{\prime} y_{2}+k_{1}^{\prime \prime} y_{3}\right) x_{1}=\frac{h}{\beta_{1}} .
$$

Now the equilibria exist when the two relations (3.6) and (3.7) are satisfied simultaneously and equilibria points are the points of intersection of the graphs of equations (3.6) and (3.7). Whenever $h=0, y_{2}=y_{3}=0$ and $x_{2}=x_{3}=0$ and this is characterized by the disease free equilibrium. If $h \neq 0$, i.e., when disease is present, then following [2] we obtain the condition for an equilibrium as

where

$$
R(h)=1
$$

$$
\begin{aligned}
R(h) & =\left[\frac{\beta_{1}\left\{k_{1}^{\prime}\left(\mu_{2}+f_{2}(h)\right)+k_{1}^{\prime \prime} \gamma_{2}\right\}}{\left(\mu_{2}+f_{2}(h)\right)\left(\mu_{2}+\gamma_{2}\right)+h\left(\mu_{2}+f_{2}(h)+\gamma_{2}\right)}\right] \\
\times & {\left[\frac{\left(\mu_{1}+\gamma_{1}\right)\left(\mu_{1}+f_{1}(h)\right)}{\left(\mu_{1}+f_{1}(h)\right)\left(\mu_{1}+\gamma_{1}\right)+h\left(\mu_{1}+f_{1}(h)+\gamma_{1}\right)}\right] }
\end{aligned}
$$

which contains the dynamics of both the systems, man and reservoir populations in contrast to Aron's model where only one system (only human population) was considered. Each of the bracketed terms in RHS of (3.9) is similar to equation (3.6) of Aron [2] and hence as shown by him is a decreasing function of $h$. Thus in our case also $R(h)$ is a decreasing function in $h$.

If $R(0)>1$, a unique equilibrium exists with disease present. If $R(0)<1$, the only equilibrium is the disease-free state. Thus $R(0)$ is the basic factor which determines the qualitative dynamics of the model. If $R(0)<1$, the disease-free equilibrium (zero equilibrium) is locally stable (appendix A) and there is no other equilibrium. On the other hand if $R(0)>1$, the zero equilibrium is unstable (appendix B). Thus $R(0)$ is the number of cases of infection in human susceptibles generated by a single infective individual in the reservoir populations through mosquito bite. In other words, $R(0)$ is the basic reproductive rate in the model. The disease will be present in the human population when $R(0)>1$.

Now

$$
R(0)=\frac{\beta_{1}\left\{k_{1}^{\prime}\left(f_{2}(0)+\mu_{2}\right)+k_{1}^{\prime \prime} \gamma_{2}\right\}}{\left(\mu_{2}+f_{2}(0)\right)\left(\mu_{2}+\gamma_{2}\right)}
$$

where

Again

$$
f_{2}(0)=\lim _{h \rightarrow 0} f_{2}(h)=\lim _{h \rightarrow 0} \frac{\left(h+\mu_{2}\right) e^{-\left(h+\mu_{2}\right) \tau_{2}}}{1-e^{-\left(h+\mu_{2}\right) \tau_{2}}} .
$$

where

$$
R(0)=\frac{\beta_{1} k_{1}^{\prime}}{\mu_{2}+\gamma_{2}}+\frac{\beta_{1} k_{1}^{\prime \prime}}{\left(\mu_{2}+f_{2}(0)\right)\left(\mu_{2}+\gamma_{2}\right)}
$$

$\frac{\beta_{1} k_{1}^{\prime}}{\mu_{2}+\gamma_{2}}=$ transmission from the infected reservoir to the human population, $\frac{\beta_{1} k_{1}^{\prime \prime}}{\mu_{2}+f_{2}(0)}=$ period of unboosted immunity in human,

and

$$
\frac{\gamma_{2}}{\mu_{2}+\gamma_{2}}=\underset{\text { probability of surviving period of infective state of the reservoirs to become }}{\text { immune. }}
$$


Figure 1 clearly demonstrates the condition for existence of the non-zero equilibrium. If $R(0)>1$, the slope of the curve (1) exceeds the slope of the line (2) at the beginning, and after a certain value of $h$, the latter exceeds the former, so that the two curves intersect two times, once at the origin (disease free state) and secondly at a point in the positive orthant.

\section{CONTROL OF THE DISEASE.}

Since J.E. is a communicable disease, it can be controlled by two ways - (i) by reducing transmission $\beta_{1}$ which can be achieved by controlling the vector populations (mosquitoes) and (ii) by immunizing the human susceptibles and gradually increasing the proportion of coverage of vaccination $(\mathrm{v})$. The ultimate goal is to reduce $R(0)$ so that $R(0)<1$ which will result in eradication of the disease. Let $\beta_{1 c}$ and $v_{c}$ be the threshold values for transmission rate and vaccination coverages respectively where $\beta_{1 c}$ and $v_{c}$ are determined by Aron [1] and Anderson and May [3].

$$
\beta_{1 c}\left(1-v_{c}\right)=\frac{k_{1}^{\prime}\left(\mu_{2}+f_{2}(0)\right)+\gamma_{2} k_{1}^{\prime \prime}}{\left(\mu_{2}+\gamma_{2}\right)\left(\mu_{2}+f_{2}(0)\right)}=1
$$

where for eradication the conditions required to be satisfied are $v>v_{c}$ and $\beta_{1}>\beta_{1 c}$. Table 1 shows the effect of reduction of transmission on the level of vaccination. It can be observed that increasing the level of vaccination in human means that less effort for reduction of transmission is required to eradicate the disease.

Now, if the infectivity $k_{1}^{\prime}$ is constant, then increasing the infectivity of the immune reservoir population (carrier) does not in contrast to Aron's findings, significantly increase the equilibrium levels of infection (Figure 2). The dynamics of J.E. spread is thus qualitatively different from that of the malaria epidemic and eventually poses less difficulty in eradication by vaccination.

Figure 3 shows the two curves for different combination of values for $k_{1}^{\prime}$ and $k_{1}^{\prime \prime}$ do not intersect at a non-zero point and, in fact, each curve is a multiple of another. This implies that, in J.E. loss of immunity $\left(f_{1}\right)$ in man is independent of the exposure rate. This is also a characteristic property of J.E., in contrast to the model given by Aron. Thus from our results (Figure 3), it indicates that boostering of immunity against J.E. is feasible only by passive immunization, i.e., direct administration of J.E. antibody in man at recurrent intervals. Acquired immunity by the attack of the disease does not persist for a long time by continued exposure to the bites of infected mosquitoes.

\section{CONCLUSION.}

The J.E. model presented here is an extension of the SIRS model by coupling the dynamics of the disease in two populations, the reservoir and the human populations. The reservoir population does not itself show any pathological symptom of the disease but acts as an intermediate host medium to pass over the infection to man through a vector population (mosquito). Infection of J.E. cannot spread from man to man or to any other animals, that is to say, man is the dead end of infection.

We have assumed that the effective reservoir of infection $\left(h_{1}\right)$ for man is proportional to the proportion of human susceptibles. The higher (lesser) is the proportion of susceptibles in a human population, the higher (lesser) is the effective reservoir of infection. In other words, in a human population where the number of susceptibles is zero, the effective reservoir of infection will be eventually nil.

It is also assumed, as in Aron ([1] and [2]), that immunes are no more infective than those who are infected both in the reservoir and human populations. The dynamic model of J.E. 
spread in reservoir population is same as that of Aron. The reservoir system is also independent of the human system but not the reverse. Aron's system has a stable disease-free equilibrium $\left(h=0, y_{2}=0, y_{3}=0\right)$ if $R(0)<1$ and a non-zero equilibrium (disease present) if $R(0)>1$. Substituting this result in the coupled system we have obtained similar results on stability properties of J.E. spread in disease by vaccination. In contrast to Aron's results we have observed that in J.E. the loss of immunity in man is independent of the rate of exposure to the disease. This implies that active immunization (direct administration of antigen in the form of live attenuated virus) does not give immunity or prolong acquired immunity in man. Vaccination by passive immunization (i.e., direct administration of serum containing antibody to man) at fixed intervals, on the other hand, will ensure control of the disease.

\section{APPENDIX A. STABILITY OF ZERO EQUILIBRIUM.}

Linearizing the system about the zero equilibrium $\left(x_{2}=0, x_{3}=0, y_{2}=0, y_{3}=0\right)$, we get the biquadratic characteristic equation

$$
\left(\lambda^{2}+\theta_{1} \lambda+\xi_{1}\right)\left(\lambda^{2}+\theta_{2} \lambda+\xi_{2}\right)=0
$$

where

$$
\begin{gathered}
\theta_{1}=2 \mu_{1}+\gamma_{1}+h+f_{1}(h) \\
\xi_{1}=\left(\mu_{1}+\gamma_{1}+h\right)\left(\mu_{1}+f_{1}(h)\right) \\
\theta_{2}=2 \mu_{2}+\gamma_{2}-\beta_{2} k_{2}^{\prime}+h+f_{2}(h) \\
\xi_{2}=\left(\mu_{2}+f_{2}(h)\right)\left(\mu_{2}+\gamma_{2}+h-\beta_{2} k_{2}^{\prime}\right)-\gamma_{2}\left(\beta_{2} k_{2}^{\prime \prime}-h\right)
\end{gathered}
$$

The roots of (A.1) having negative real parts, the zero equilibrium is locally stable if and only if $\theta_{1}, \xi_{1}, \theta_{2}$ and $\xi_{2}$ are all positive.

Now $\theta_{1}$ and $\xi_{1}$ are always positive. The condition that $\xi_{2}>0 \Leftrightarrow R(0)<1$, where $R(0)$ is given in equation (3.10). Again $R(0)<1 \Rightarrow \theta_{2}>0$. Hence if $R(0)>1$, the zero equilibrium is locally unstable.

\section{APPENDIX B. STABILITY OF NONZERO EQUILIBRIUM.}

Linearizing the system about the non-zero equilibrium $\left(x_{2}, x_{3}, y_{2}\right.$ and $\left.y_{3}\right)$, we get the biquadratic characteristic equation

$$
\left(\lambda_{2}+\alpha_{1} \lambda+\eta_{1}\right)\left(\lambda^{2}+\alpha_{2} \lambda+\eta_{2}\right)=0
$$

where

$$
\begin{gathered}
\alpha_{1}=2 \mu_{1}+\gamma_{1}+h+f_{1}(h)+\beta_{1}\left(k_{1}^{\prime} y_{2}+k_{1}^{\prime \prime} y_{3}\right)\left(1-x_{2}-x_{3}\right)-\beta_{1} f_{1}^{\prime}(h)\left(k_{1}^{\prime} y_{2}+k_{1}^{\prime \prime} y_{3}\right) x_{3} \\
\eta_{1}=\left\{\mu_{1}+\gamma_{1}+h+\beta_{1}\left(k_{1}^{\prime} y_{2}+k_{1}^{\prime \prime}\right)\left(1-x_{2}-x_{3}\right)\right\}\left\{\mu_{1}+f_{1}(h)-\beta_{1} f_{1}^{\prime}(h)\left(k_{1}^{\prime} y_{2}+k_{1}^{\prime \prime} y_{3}\right) x_{3}\right\} \\
+\left\{\gamma_{1}+\beta_{1} f_{1}^{\prime}(h)\left(k_{1}^{\prime} y_{2}+k_{1}^{\prime \prime} y_{3}\right) x_{3}\right\}\left\{h+\beta_{1}\left(k_{1}^{\prime} y_{2}+k_{1}^{\prime \prime} y_{3}\right)\left(1-x_{2}-x_{3}\right)\right\} \\
\alpha_{2}=2 \mu_{2}+\gamma_{2}+h+f_{2}(h)+\beta_{2} f_{2}^{\prime}(h) k_{2}^{\prime \prime} y_{3}-\beta_{2} k_{2}^{\prime}\left(1-y_{2}-y_{3}\right) \\
\eta_{2}=\left\{\mu_{2}+\gamma_{2}+h-\beta_{2} k_{2}^{\prime}\left(1-y_{2}-y_{3}\right)\right\}\left\{\mu_{2}+f_{2}(h)+\beta_{2} f_{2}^{\prime}(h) k_{2}^{\prime \prime} y_{3}\right\} \\
+\left(\gamma_{2}-\beta_{2} f_{2}^{\prime}(h) k_{2}^{\prime} y_{3}\right)\left(h-\beta_{2} k_{2}^{\prime \prime}\left(1-y_{2}-y_{3}\right)\right) .
\end{gathered}
$$

We see that the equilibrium rate of exposure $h$ is the function of $x_{1}, y_{2}$ and $y_{3}$ as defined in equation (2.2) and (2.3). The roots of equation (B.1) having negative real parts, the non-zero equilibrium is locally stable if and only if all $\alpha_{1}, \eta_{1}, \alpha_{2}$ and $\eta_{2}$ are positive.

We note that, $\alpha_{1}>0$ always, since $-1<f_{1}^{\prime}(h)<0$. 
After canceling few common terms $\eta_{1}$ may be written as

$$
\begin{gathered}
\eta_{1}=-\left(\mu_{1}+\gamma_{1}\right) \beta_{1} f_{1}^{\prime}(h)\left(k_{1}^{\prime} y_{2}+k_{1}^{\prime \prime} y_{3}\right) x_{3}+\left(\mu_{1}+f_{1}(h)\right) \beta_{1}\left(k_{1}^{\prime} y_{2}+k_{1}^{\prime \prime} y_{3}\right)\left(1-x_{2}-x_{3}\right) \\
+\gamma_{1} \beta_{1}\left(k_{1}^{\prime} y_{2}+k_{1}^{\prime \prime} y_{3}\right)\left(1-x_{2}-x_{3}\right)+\left(\mu_{1}+f_{1}(h)\right)\left(\mu_{1}+\gamma_{1}+h\right)+\gamma_{1} h .
\end{gathered}
$$

Therefore

$$
\eta_{1}>0\left[\text { as }-1<f_{1}^{\prime}(h)<0\right]
$$

From (2.3), (2.7) and $f_{2}^{\prime}(h)>-1$ we have

$$
h>\beta_{2} k_{2}^{\prime \prime} y_{3}>\beta_{2} k_{2}^{\prime \prime} f_{2}^{\prime}(h) y_{3}
$$

Again from (3.1), (3.2), (2.3) and (2.7) we have

$$
\beta_{2} k_{2}^{\prime}\left(1-y_{2}-y_{3}\right)=\frac{\beta_{2} k_{2}^{\prime} y_{2}\left(\mu_{2}+\gamma_{2}\right)}{\beta_{2}\left(k_{2}^{\prime} y_{2}+k_{2}^{\prime \prime} y_{3}\right)}<\mu_{2}+\gamma_{2}
$$

$\alpha_{2}>0$, always holds when a non-zero equilibrium exists.

$\eta_{2}$ may be written as

$$
\begin{aligned}
\eta_{2}=\left(\mu_{2}+\gamma_{2}+\right. & \left.h-\beta_{2} k_{2}^{\prime}\left(1-y_{2}-y_{3}\right)\right) \beta_{2} f_{2}^{\prime}(h) k_{2}^{\prime \prime} y_{3} \\
& +\beta_{2} k_{2}^{\prime}\left(y_{2}+y_{3}\right)\left(\mu_{2}+f_{2}(h)\right)+\beta_{2} f_{2}^{\prime}(h) k_{2}^{\prime} y_{3} \beta_{2} k_{2}^{\prime \prime}\left(1-y_{2}-y_{3}\right) \\
& -h \beta_{2} f_{2}^{\prime}(h) k_{2}^{\prime} y_{3}+\left\{h \gamma_{2}-\gamma_{2} \beta_{2} k_{2}^{\prime \prime}+\left(\mu_{2}+\gamma_{2}+h\right)\left(\mu_{2}+f_{2}(h)\right)\right. \\
& \left.-\beta_{2} k_{2}^{\prime}\left(\mu_{2}+f_{2}(h)\right)\right\}+\gamma_{2} \beta_{2} k_{2}^{\prime \prime}\left(y_{2}+y_{3}\right)
\end{aligned}
$$

Since the equilibrium point must satisfy (3.8) which again implies

$$
\frac{\beta_{2}\left\{k_{2}^{\prime}\left(\mu_{2}+f_{2}(h)\right)+k_{2}^{\prime \prime} \gamma_{2}\right\}}{\left(\mu_{2}+\gamma_{2}\right)\left(\mu_{2}+f_{2}(h)\right)+h\left(\mu_{2}+\gamma_{2}+f_{2}(h)\right)}=1
$$

the term within curly brackets is zero.

Again, from (3.1) and (3.2) we have

$$
\gamma_{2}\left(y_{2}+y_{3}\right)=y_{3}\left(\mu_{2}+\gamma_{2}+f_{2}(h)\right) .
$$

The remaining terms in (B.8) may be written as

$$
\begin{gathered}
-h \beta_{2} f_{2}^{\prime}(h) y_{3}\left(k_{2}^{\prime}-k_{2}^{\prime \prime}\right)+\beta_{2} k_{2}^{\prime \prime} y_{3}\left(\gamma_{2}+\mu_{2}\right)\left\{\left(1+f_{2}^{\prime}(h)+f_{2}(h)\right\}\right. \\
+\beta_{2} k_{2}^{\prime}\left(y_{2}+y_{3}\right)\left(\mu_{2}+f_{2}(h)\right) \\
+\beta_{2} f_{2}^{\prime}(h) k_{2}^{\prime} y_{3}\left(1-y_{2}-y_{3}\right) \beta_{2} k_{2}^{\prime \prime}-\beta_{2} k_{2}^{\prime}\left(1-y_{2}-y_{3}\right) \beta_{2} f_{2}^{\prime}(h) k_{2}^{\prime \prime} y_{3}
\end{gathered}
$$

The last two terms in (B.9) cancel each other and therefore $\eta_{2}>0$ since, $k_{2}^{\prime}>k_{2}^{\prime \prime}$ and $-1<f_{2}^{\prime}(h)<0$. Thus whenever the non-zero equilibrium exists, it is stable. 


\section{TABLE 1}

Eradication criteria for J.E.

Level of vaccination

$100 v_{c}$

$0 \%$

$25 \%$

$50 \%$

$75 \%$

$80 \%$

$85 \%$
Reduction of Transmission

$100\left(1-\frac{\beta_{1 c}}{\beta_{1}}\right)$

$93.9 \%$

$89.9 \%$

$81.9 \%$

$57.9 \%$

$45.9 \%$

$25.9 \%$

The eradication criteria are given in (4.1).

The parameters used are $\beta_{1}=1, \mu_{2}=0.02, \tau=1, \gamma_{2}=0.1, k_{2}^{\prime}=1, k_{2}^{\prime \prime}=0.6, f_{2}(0)=0.99$.

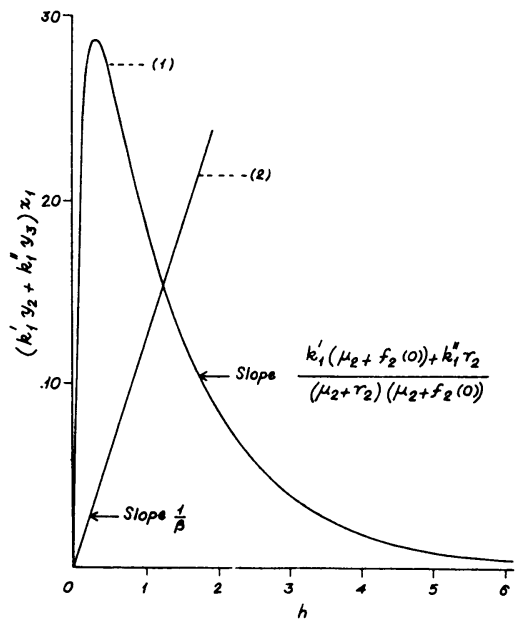

Fig.1 Effective reservoir of infection in man, $\left(k_{1}^{\prime} y_{2}+k_{1}^{\prime \prime} y_{3}\right) x_{1}$ as a function of the rate of exposure $h$, according to (1) equation (3.6) and (2) equation (3.7). The parameters used are $B_{1}=8, \mu_{1}=0.02$, $\mu_{2}=0.2, \gamma_{1}=0.7, \gamma_{2}=0.1, \tau_{1}=\tau_{2}=1, k_{1}^{\prime}=1, k_{1}^{\prime \prime}=0.5$ 


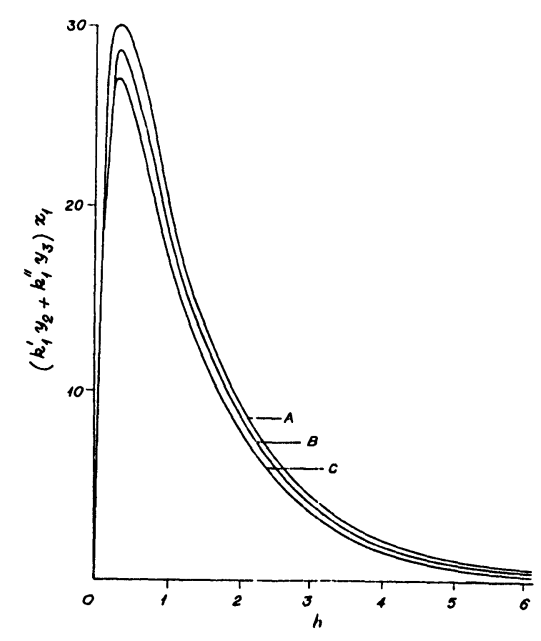

Fig.2 Effective reservoir of infection in man, $\left(k_{1}^{\prime} y_{2}+k_{1}^{\prime \prime} y_{3}\right) x_{1}$ as a function of the rate of exposure $h$, according to equation (3.6): (A) $k_{1}^{\prime \prime}=1$, (B) $k_{1}^{\prime \prime}=0.5$, (C) $k_{1}^{\prime \prime}=0$. The rest of the parameters used are $\mu_{1}=0.02, \mu_{2}=0.2, \gamma_{1}=0.7, \gamma_{2}=0.1, \tau_{1}=\tau_{2}=1, \quad k_{1}^{\prime}=1$.

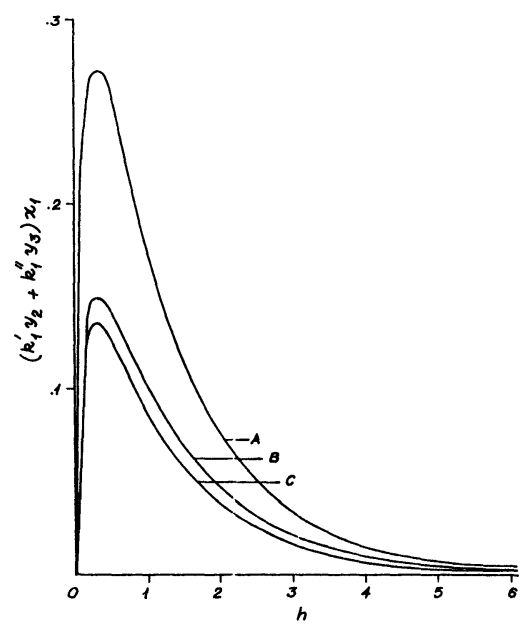

Fig.3 Effective reservoir of infection in man, $\left(k_{1}^{\prime} y_{2}+k_{1}^{\prime \prime} y_{3}\right) x_{1}$ as a function of the rate of exposure $b$ according to equation (3.6): (A) $k_{1}^{\prime}=1$, $k_{1}^{\prime \prime}=0$, (B) $k_{1}^{\prime}=0.5, k_{1}^{\prime \prime}:=0.5$, (C) $k_{1}=0.5, k_{1}^{\prime \prime}=0$. The rest of the parameters used are $\mu_{1}=0.02, \mu_{2}=0.2, \gamma_{1}=0.7$, $\gamma_{2}=0.1, \tau_{1}=\tau_{2}=1$.

\section{REFERENCES}

1. ARON, J.L. Dynamics of acquired immunity boosted by exposure to infection, Math. Biosci., 64 (1983), 249-259.

2. ARON, J.L. Acquired Immunity Dependent upon exposure in an. SIRS epide mic model, Math. Bi(ısci., 88 (1988), 37-47.

3. ANDERSON, R.M. AND MAY, R.M. Directly transmitted infectious diseases: Control by vaccirlation, Science 215 (1982), 1053-1060. 


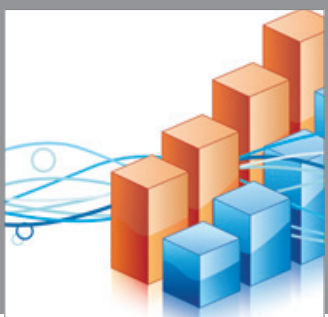

Advances in

Operations Research

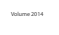

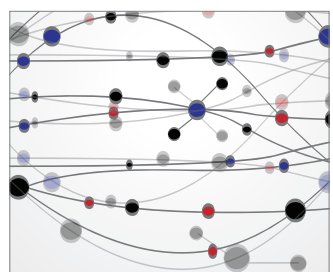

\section{The Scientific} World Journal
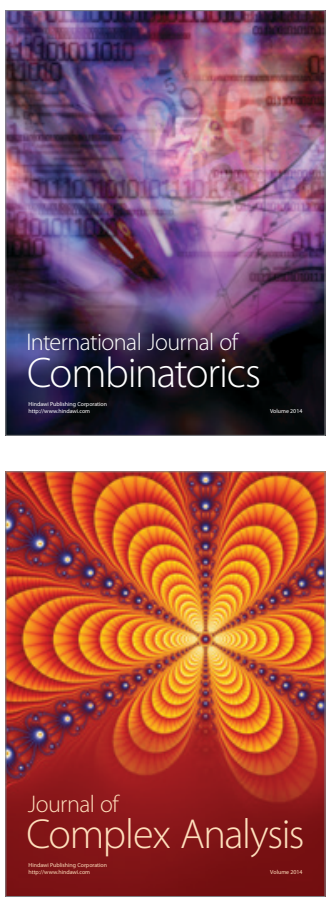

International Journal of

Mathematics and

Mathematical

Sciences
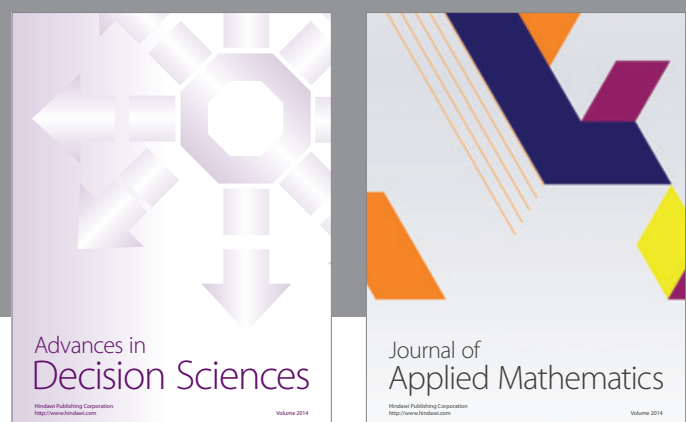

Journal of

Applied Mathematics
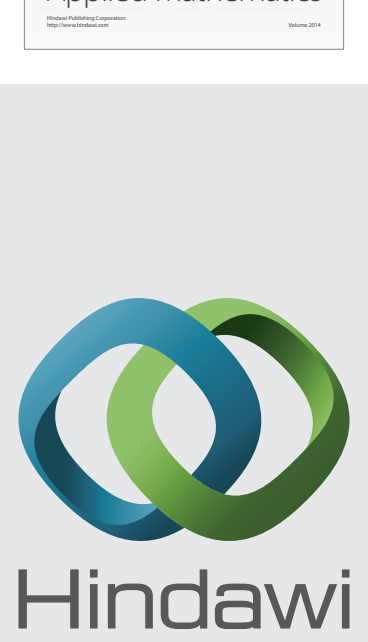

Submit your manuscripts at http://www.hindawi.com
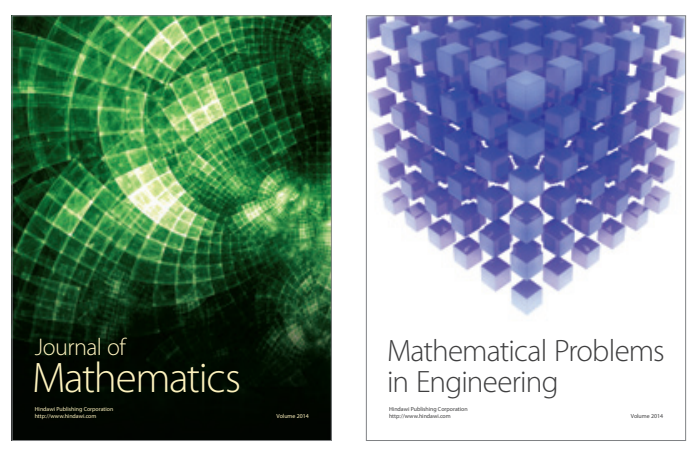

Mathematical Problems in Engineering
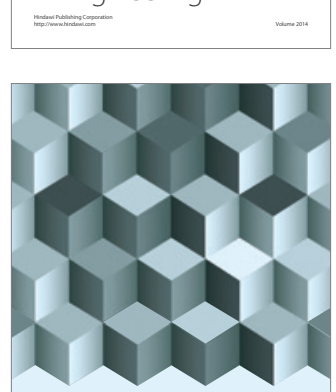

Journal of

Function Spaces
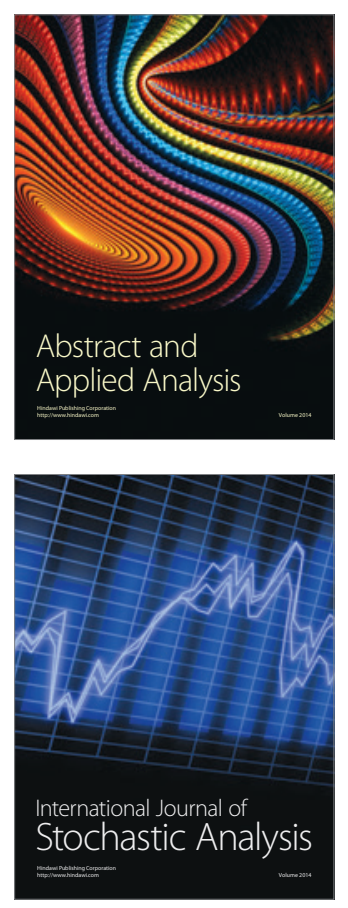

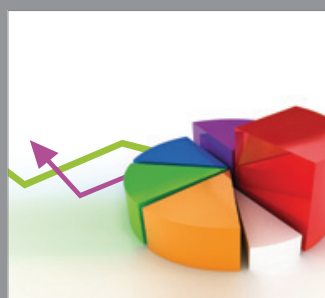

ournal of

Probability and Statistics

Promensencen
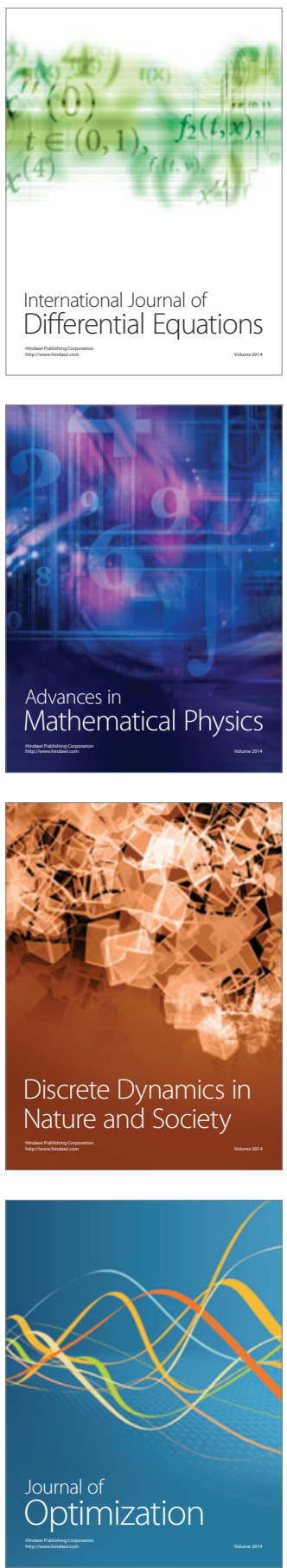